SHORT REPORT

\title{
Retinal abnormalities in CADASIL: a retrospective study of 18 patients
}

\author{
R Cumurciuc, P Massin, M Pâques, V Krisovic, A Gaudric, M G Bousser, H Chabriat
}

J Neurol Neurosurg Psychiatry 2004;75:1058-1060. doi: 10.1136/jnnp.2003.024307

\begin{abstract}
Background: CADASIL is an inherited small vessel disease related to Notch3 gene mutations.

Aim: To report retinal findings in symptomatic CADASIL patients.

Methods: Assessment of visual acuity (VA), testing of visual fields (VF), funduscopic examination (FE), and fluorescein angiography (FA) were carried out in 18 symptomatic patients.

Results: No visual symptoms were presented by our patients. VA was normal in all. Ophthalmologic abnormalities were found in 8 patients. VF were normal except for a right hemianopia in one subject due to ischemic stroke. FE and FA revealed significant abnormalities in seven other subjects (mean age: 55 years; range: $39-74)$ : nerve fibre loss $(n=4)$, cotton wool spots $(n=3)$, sheathed arteries $(n=1)$, and tortuous arteries $(n=1)$. Only one patient with both tortuous arteries and nerve fibre loss had multiple vascular risk factors, and another patient with cotton wool spots was a current smoker.

Discussion: FE and FA revealed silent retinal abnormalities in CADASIL patients with nerve fibre loss in $22 \%$ and cotton wool spots in $17 \%$. The presence of these abnormal retinal findings does not seem related to the severity of the disorder but may be considered as peripheral markers of this genetic disease.
\end{abstract}

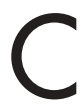
erebral autosomal dominant arteriopathy with subcortical infarcts and leukoencephalopathy (CADASIL) is an inherited small artery disease related to Notch3 gene mutations on chromosome 19. Although CADASIL is a systemic vascular disease, its clinical expression is purely neurological and is characterised mainly by subcortical infarcts and dementia and less frequently by migraine with aura and mood disorders. It starts in mid-adulthood and leads to death in a mean of 20 years. White matter lesions as seen on MRI T2 or FLAIR sequences are present in all patients.

CADASIL is distinct from atherosclerotic and amyloid angiopathies. One hallmark of the disease is the presence of granular osmiophilic material (GOM) seen on electron microscopy and associated with degenerated smooth muscle cells within the walls of leptomeningeal and medullary arteries of the brain. Similar lesions have been reported in the spinal cord and in other organs such as the spleen, heart, liver, small intestine, and muscles. ${ }^{1}$ The detection of such typical vascular alterations ${ }^{2}$ and the presence of an abnormal Notch3 immunostaining in skin biopsies is now used for diagnosis. ${ }^{3}$

Ruchoux et al reported these typical vascular wall alterations in retinal arteries in a single post mortem case of CADASIL. ${ }^{1}$ However, details of ophthalmologic examination in CADASIL patients are scarce. Robinson et al reported the case of a 45 year old patient with visual field abnormalities associated with attenuated and sheathed vessels at funduscopic examination and patchy hyperfluorescence of the retina at fluorescein angiography. ${ }^{4}$ In an abstract Uchino et al recently reported a marked narrowing of retinal vessels in 3/6 subjects with CADASIL and CADASIL like disorders. ${ }^{5}$

Herein, we report the results of detailed ophthalmologic examination including evaluation of visual acuity and visual field, and fundoscopic photography and fluorescein angiography in a series of 18 CADASIL patients.

\section{METHODS}

Eighteen symptomatic patients over 18 years of age and having different mutations of the Notch3 gene responsible for CADASIL were included in this study.

The main clinical manifestations are summarised in table 1. Eleven patients had a history of completed stroke and six were demented according to DSM-IV criteria. None of our patients complained of visual symptoms. All patients had white matter abnormalities on MRI which were rated as grade B (punctiform or slight periventricular, or both) in one patient, grade $\mathrm{C}$ (nodular or moderate periventricular, or both) in six, and grade D (confluent lesions or severe periventricular, or both) in $11 .^{6}$ Vascular risk factors were present in 13 patients, of whom nine had dyslipidemia, five were current smokers, one was being treated for hypertension, and one was diabetic.

Ophthalmologic examination included: (a) assessment of visual acuity and fundoscopic examination; and (b) detection of visual field defects using Goldmann perimetry $(\mathrm{n}=2)$ or the Humphrey Field analyser $(n=12)$. Four patients did not undergo visual field examination. Photographs of the fundus were taken in all patients and all but one patient had a fluorescein angiography.

The ophthalmologic data and charts were reviewed by two ophthalmologists blinded to the subject's clinical manifestations of CADASIL. The following parameters were systematically assessed in each case and were recorded after agreement of the two observers: the presence or absence of visual field defects on the perimetry charts, vascular calibre and fundus appearance (both visually evaluated), and the presence of microvascular retinal abnormalities such as micro aneurysms, retinal haemorrhages, cotton wool spots or retinal hard exudates, and optic disk abnormalities.

The data obtained in this study were collected through a research project approved by the ethics committee of PitieSalpetriere hospital. All subjects gave their consent for the ophthalmologic examination.

Abbreviations: CADASIL, cerebral autosomal dominant arteriopathy with subcortical infarcts and leukoencephalopathy; FA, fluorescein angiography; $\mathrm{FE}$, funduscopic examination; $\mathrm{GOM}$, granular osmiophilic material; $V A$, visual acuity; $V F$, visual fields 
Table 1 Baseline characteristics and main clinical manifestations and retinal abnormalities of CADASIL patients

\begin{tabular}{|c|c|c|c|}
\hline & All, $n=18$ & $\begin{array}{l}\text { Positive retinal } \\
\text { findings, } n=7 \text { (39\%) }\end{array}$ & $\begin{array}{l}\text { Negative retinal findings, } \\
n=11(61 \%)\end{array}$ \\
\hline Age (years) & 56 (range: $39-74$ ) & 55 (range: $39-74$ ) & 59 (range: $41-70$ ) \\
\hline Migraine $(n)$ & $7(39 \%)$ & 2 & 5 \\
\hline Isolated aura (n) & $2(11 \%)$ & 2 & 0 \\
\hline Mood disturbances (n) & $7(39 \%)$ & 3 & 4 \\
\hline Ischemic events (n) & $13(72 \%)$ & 4 & 9 \\
\hline TIAs (n) & 7 & 2 & 5 \\
\hline Completed strokes (n) & 11 & 4 & 7 \\
\hline Dementia (n) & $6(33 \%)$ & 1 & 5 \\
\hline \multicolumn{4}{|c|}{ White matter abnormalities } \\
\hline Grade B (n) & 1 & 0 & 1 \\
\hline Grade C (n) & 6 & 4 & 2 \\
\hline Grade D (n) & 11 & 3 & 8 \\
\hline \multicolumn{4}{|l|}{ Retinal abnormalities } \\
\hline Nerve fibre loss (n) & $4(22 \%)$ & & \\
\hline Mean age (years) & 56 & & \\
\hline Cotton wool spots (n) & $3(17 \%)$ & & \\
\hline Mean age (years) & 50 & & \\
\hline Sheathed arteries ( $n$ ) & $1(5.5 \%)$ & & \\
\hline Tortuous arteries ( $\mathrm{n}$ ) & $1(5.5 \%)$ & & \\
\hline
\end{tabular}

\section{RESULTS}

Visual acuity (with or without correction) was normal in all patients.

Retinal abnormalities were found in seven patients (mean age: 55 years; range: 39-74) (table 1). Funduscopic examination and fluorescein angiography revealed: cotton wool spots $(\mathrm{n}=3)$ (fig $1 \mathrm{~A}$ and $\mathrm{B})$, isolated nerve fibre loss $(\mathrm{n}=2)$ (fig 1C), and nerve fibre loss associated with sheathed arteries in one patient (fig 1D) and with tortuous arteries in another.

Of the 14 patients who had a visual field examination, only one had abnormal results related to a right hemianopia secondary to an ischemic stroke in the left middle cerebral artery territory. This patient had normal funduscopic examination and fluorescein angiography.

Vascular risk factors were present in two of the seven patients with retinal abnormalities. One subject who had both tortuous arteries and nerve fibre loss was a current smoker and was being treated for diabetes (type II). Another subject who presented with cotton wool spots was also a current smoker. Four of the seven patients with abnormal retinal findings had a past history of completed stroke and one was demented.

The frequency of the main symptoms of the disease did not differ between patients with and without abnormal retinal findings. In addition, the severity of white matter signal abnormalities did not differ between these two groups $\left(\chi^{2}\right.$ test, $\mathrm{p}=0.2$; table 1 ).

\section{DISCUSSION}

Some $39 \%$ of symptomatic CADASIL patients presented with abnormal retinal findings. The most frequent retinal changes included cotton wool spots (17\%) and retinal nerve fibre layer defects $(22 \%)$ suggesting underlying circulatory disturbances.

Cotton wool spots are presumably related to localised accumulations of axoplasmic debris in the retinal nerve fibre layer and may result from interruption of axoplasmic transport. They are considered to be local consequences of arteriolar obliteration and usually disappear within weeks after the ischemic event without any ophthalmologic consequences. They are frequent in patients with diabetes mellitus, hypertension, or arteriosclerosis. However, patients harbouring these retinal abnormalities in the present study had no vascular risk factors except for tobacco smoking in a single case, which suggests that such retinal lesions are indeed manifestations of CADASIL.

An association between retinal exudates and the presence of lacunar infarcts has been already reported by Kwa et al in patients with small vessel diseases. ${ }^{7}$ Herein, two of the three patients with cotton wool spots had a history of lacunar infarcts, but seven patients who also had lacunar lesions did not have any retinal abnormalities. In addition, we found no differences in the neurological presentation or in the extent of MRI lesions between patients with and without retinal cotton wool spots, which suggests that these retinal findings are not related to the severity of the disease at the cerebral level in CADASIL.

Alterations of axoplasmic transport may also lead to physical alterations of retinal nerve fibre layers resulting in some cases in sector atrophy, as observed in four patients. Such defects are observed after local ischemia but may also result from chronic glaucoma or remote damage of the optic nerve, chiasma, or optic tracts, which was not the case in our patients. The mean age of patients with nerve fibre defects was slightly higher than that of patients presenting with cotton wool spots ( $56 v 50$ years), suggesting that nerve fibre layer defects may be sequelae of previous cotton wool spots in these patients.

Isolated retinal fibre deficit was found in two patients without cardiovascular risk factor, and may be considered as actual manifestations of CADASIL. In another patient with no cardiovascular risk factors, retinal nerve fibre layer defects were associated with arterial sheathing. This patient had severe white matter lesions on MRI similar to the patient reported by Robinson et al. ${ }^{4}$ Arterial sheathing is thought to result from increased optical density of the vessel wall in advanced sclerosis with a marked increase and disarrangement of collagen fibrils in the media and adventitia. ${ }^{8}$ Whether this retinal aspect is observed only in advanced stages of CADASIL warrants further investigations.

In contrast the only patient who had retinal nerve fibre deficit and tortuous arteries presented with multiple vascular risk factors including diabetes, dyslipidemia, and tobacco smoking and had diffuse atheromatosis of the main cervical arteries. The retinal findings in this case are possibly related to the association of vascular risk factors and CADASIL.

Recently Parisi et al reported electrophysiologic abnormalities of the retinal layers in asymptomatic CADASIL patients. ${ }^{9}$ 

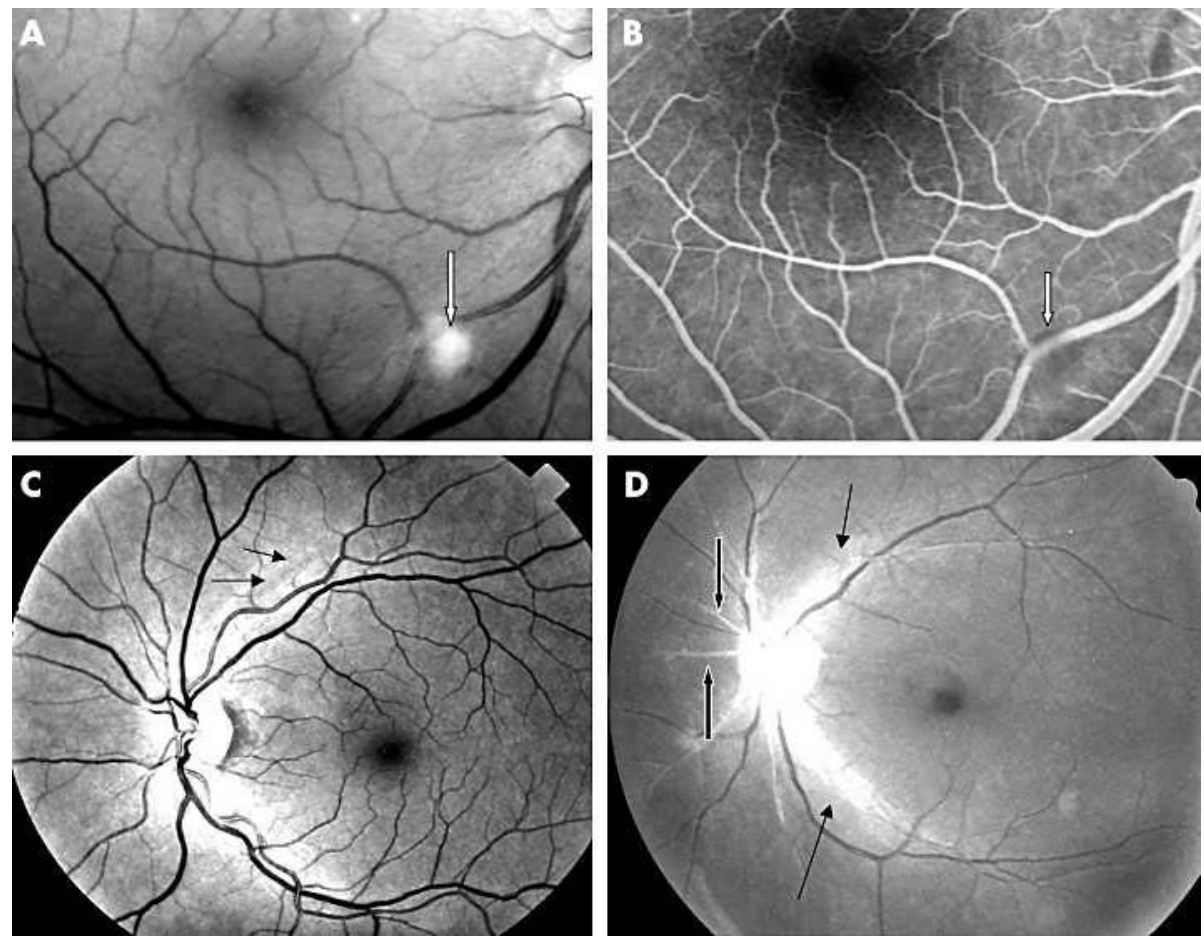

Figure 1 Fundus photography (green filter) (A) and fluorescein angiography (B) showing cotton wool spots in a CADASIL patient: a large spot (arrow) is observed at funduscopy. This cotton wool spot attenuates the appearance of the vessel lumen on angiography (B). (C) Retinal photography showing nerve fibre loss in a CADASIL patient (arrows). (D) Retinal photography showing arterial sheathing (large arrows) associated with retinal nerve fibre loss (small arrows) in a CADASIL patient.

In the present study, we did not use electroretinography to detect these silent functional abnormalities which may precede the retinal abnormalities observed in our patients. Whether these abnormal electric responses at the retinal level are related to circulatory disturbances occurring at the capillary level or to more severe lesions as reported in this study remains to be investigated.

In conclusion, retinal abnormalities are not rare in CADASIL and are presumably related to the alterations of small retinal vessels already observed postmortem. They are clinically silent and overall do not seem to be related to the severity of the disorder at the cerebral level but may be considered as peripheral markers of this genetic disorder.

\section{Authors' affiliations \\ R Cumurciuc, M G Bousser, H Chabriat, Department of Neurology, Lariboisiere Hospital, 2, rue A Paré, 75010 Paris, France P Massin, M Pâques, V Krisovic, A Gaudric, Department of Ophthalmology, Lariboisiere Hospital, 2, rue A Paré, 75010 Paris, France \\ Competing interests: none declared}

Correspondence to: Professor H Chabriat, Department of Neurology, Lariboisiere Hospital, 2, rue Ambroise Paré, 75010 Paris, France; hugues.chabriat@|rb.ap-hop-paris.fr
Received 5 August 2003

In revised form 5 November 2003

Accepted 6 November 2003

\section{REFERENCES}

1 Ruchoux MM, Guerouaou D, Vandenhaute B. Systemic vascular smooth muscle cell impairment in cerebral autosomal dominant arteriopathy with subcortical infarcts and leukoencephalopathy. Acta Neuropathol (Berl) 1995:89:500-12.

2 Ruchoux MM, Chabriat $H$, Baudrimont $M$, et al. Presence of ultrastructural arterial lesions in muscle and skin vessels of patients with CADASIL. Stroke 1995;25(11):2291-92.

3 Joutel A, Favrole $P$, Labauge $P$, et al. Skin biopsy immunostaining with a Notch3 monoclonal antibody for CADASIL diagnosis. Lancet 2001;358(9298):2049-51

4 Robinson W, Galetta SL, McCluskey L, et al. Retinal findings in cerebral autosomal dominant arteriopathy with subcortical infarcts and leukoencephalopathy (CADASIL). Surv Ophthalmol 2001;45(5):445-8.

5 Uchino M, Uyama E, Maeda Y, et al. CADASIL: clinical analysis of CADASIL and CADASIL-like disorders in Japan (abstract). Rinsho Shinkeigaku 2000;40(12):1247-50.

6 Chabriat $\mathrm{H}$, Levy $\mathrm{C}$, Taillia $\mathrm{H}$, et al. Patterns of MRI lesions in CADASIL. Neurology 1998;51:452-7.

$7 \mathrm{Kwa}$ VIH, Van der Sande JJ, Stam J, et al. Retinal arterial changes correlate with cerebral small-vessel disease. Neurology 2002;59:1536-40.

8 Iwassaki $M$, Ishibashi T, Inomata $H$, et al. Ultrastructure of sheathed vessels in the retina from patients with various diseases. Graefe's Arch Clin Exp Ophthalmol 1987;225(3):177-84.

9 Parisi V, Pierelli F, Fattapposta F, et al. Early visual function impairment in CADASIL. Neurology 2003;60:2008-10. 